\title{
Studies on the Solidification Path of Single Crystal Superalloys
}

\author{
Nils Warnken
}

(Submitted August 19, 2015; in revised form December 14, 2015; published online January 26, 2016)

\begin{abstract}
Single crystal superalloys are widely used in high temperature sections of turbines, where they are able to withstand extended exposure to high temperatures. As these alloys are commonly cast and directionally solidified via the Bridgman process, the knowledge of their solidification path is of great importance. This paper gives an overview of aspects of the solidification path in superalloys, as studied experimentally and from a modeling and simulation point of view. The implications of microsegregation, and sequence of phase formation are discussed. Attention is given to the processes leading to the formation of interdendritic $\gamma^{\prime}$, also often referred to as the $\gamma / \gamma^{\prime}$ eutectic.
\end{abstract}

Keywords microstructure, multicomponent, phase field modeling, single crystal superalloy, solidification

\section{Introduction}

What kind of material is required for applications at high temperatures? And how are parts for these applications manufactured, as most likely even higher temperatures are required during manufacture? These-possibly slightly naïve-questions arise quickly when thinking about devices such as turbines for jet engines or power generation. Interestingly metallic alloys are still very common, despite numerous efforts to introduce other materials such as ceramics, and rather traditional manufacturing routes such as casting are still very widespread. A class of alloys widely used for the hottest section of jet-engines are single crystal superalloys. ${ }^{[1]}$ These alloys are possibly among the most complex alloys ever created by man in terms of their chemical composition. It is common for them to contain around ten major alloying elements, with several minor additions. Balancing these in order to achieve the required alloy properties is a formidable task. ${ }^{[2,3]}$ The aim is to achieve a microstructure containing precipitates of the strengthening $\gamma^{\prime}$ phase in a matrix of $\gamma$ phase, the former one

This article is an invited paper selected from presentations at the Hume-Rothery Award Symposium on "Multicomponent Alloy Metallurgy, the Bridge from Materials Science to Materials Engineering," during TMS 2015, held March 15-19, 2015, in Orlando, Fla., and has been expanded from the original presentation. This symposium was held in honor of the 2015 Hume-Rothery award recipient, William Boettinger, for outstanding contributions to thermodynamics and kinetics of metallurgical systems and their application to the understanding of alloy microstructures and the relationship to processing conditions.

Nils Warnken, School of Metallurgy and Materials, University of Birmingham, Edgbaston, Birmingham B15 2TT, UK. Contact e-mail: n.warnken@bham.ac.uk. showing an ordered structure $\left(\mathrm{Ll}_{2}\right)$ while the latter one exhibits an fcc structure. Interestingly the precipitates contribute about 75 vol.\% to the microstructure, making them the majority phase. ${ }^{[4]}$ This microstructure provides a large number of obstacles to hamper dislocation movement at high temperature, thus increasing high temperature creep performance.

Sophisticated casting processes have been developed in order to improve high temperature creep properties even further. ${ }^{[1,5]}$ Grain boundaries are the first to weaken at high temperatures. Directional solidification via the Bridgman process allows either aligning the grains along the main axis of the cast part, or removing grain boundaries altogether, the latter leading to the formation of single crystals or more precisely single grains. Although single crystal superalloys form single phase microstructures during equilibrium solidification-showing only $\gamma$ phase-microsegregation leads to formation $\gamma$ dendrites and interdendritic $\gamma^{\prime}$ during technical processing. ${ }^{[6-8]}$ The segregation of alloying elements is therefore highly relevant. Chemical inhomogeneities lead to local variation of alloy properties and the formation of unwanted phases, such as topologically closed packed (TCP) phases. ${ }^{[9,10]}$ After casting single crystal superalloys are thus commonly heat treated in order to remove microsegregation, to control the size and shape of the $\gamma^{\prime}$ precipitates and hence optimize creep behavior. ${ }^{[11-14]}$ During casting and solidification itself, elemental segregation can lead to the formation of casting defects such as freckles, which destroy the single crystalline structure of the material. $^{[15-17]}$

It becomes apparent that knowledge of the solidification behavior of single crystal superalloys is important for the design of these alloys and the understanding of the manufacturing processes. This paper is concerned with the solidification path of single crystal superalloys and the consequences for as-cast microstructure formation. In this short review a number of aspects of solidification paths in these alloys will be discussed based on work published by other researchers and the authors own work and some previously unpublished material. The aim is to provide a 
wider view than would be possible in a paper presenting specialized, most recent research findings.

\section{Alloy Constitution}

The fundamental aspects of the solidification path are determined by the constitution of the alloy. The basis for single crystal superalloys is the $\mathrm{Ni}-\mathrm{Al}$ phase diagram. Interestingly early versions of the phase diagram show a eutectic reaction between the $\gamma$ and the $\gamma^{\prime}$ phase, while a number of works have demonstrated very clearly that it is a peritectic reaction. ${ }^{[18-21]}$ Despite these, the term $\gamma / \gamma^{\prime}$ eutectic is extremely common. The binary $\mathrm{Ni}-\mathrm{Al}$ phase diagram shows a peritectic reaction between the $\gamma$ and $\gamma^{\prime}$ phases and a eutectic line between the $\gamma^{\prime}$ and $\beta(\mathrm{NiAl}$, ordered bcc/B2 phase) phases. These two invariant lines are very close together, separated by just a few degrees and the differences in the relevant Gibbs energies are very small. This made an accurate determination of the nature of the $\gamma / \gamma^{\prime}$ reaction quite challenging.

With the development of advanced thermodynamic databases according to the CALPHAD method, it is possible to calculate the fraction solid versus temperature evolution and predict elemental segregation. ${ }^{[22-24]}$ Although CALPHAD databases model equilibrium conditions only, the fraction solid versus temperature evolution can be calculated using the Scheil model. This approach assumes perfect mixing of the liquid and no transport of solute in the solid. For primary and eutectic solidification this is straightforward, as solid diffusion does not play a significant role. For peritectic alloys diffusion in the solid is necessary to complete the reaction. Under Scheil conditions however, it is found that entering the peritectic reaction affects which phases form from the melt, but the disappearance of the preperitectic phase must be ignored as there is no diffusion in the solid. ${ }^{24]}$ For technical solidification of alloys with mainly substitutional elements this is still a good approximation, due to the slow diffusion in the solid phases. Is it still possible though to distinguish eutectic and peritectic reactions from fraction solid versus temperature profiles? Figure 1 compares fraction liquid versus temperature for a five component model superalloy-composition is given in Table 1-using two different databases which model liquid/ $\gamma / \gamma^{\prime}$ reaction as peritectic (top, solid and dotted line, Dupin database $^{[22]}$ ) and eutectic (bottom, TTNi7 database ${ }^{[23]}$ ). It can be seen that the onset of the reaction is barely noticeable in both cases, unlike the $\mathrm{liq} / \gamma / \mathrm{BCC}$ reaction in the top diagram, which leads to a rapid drop in the fraction liquid upon cooling. It has to be noted that BCC in this case refers to the ordered B2 phase. To this date the term $\gamma / \gamma^{\prime}$ eutectic is still very widely used in the Superalloys community. A more neutral and hence appropriate expression would be however interdendritic $\gamma^{\prime}$.

\section{Microsegregation and Solidification Sequence}

The segregation of alloying elements in single crystal superalloys has been the subject of several studies. ${ }^{[25-27]}$ The as-cast microstructure consists of $\gamma$ dendrites with interdendritic $\gamma^{\prime}$ present due to the microsegregation of alloying elements. Figure 2 shows a typical microstructure found in directionally solidified CMSX-4. The single crystal superalloys are originally designed to show a single phase $\gamma$ region below the equilibrium solidus temperature. Hence the presence of interdendritic $\gamma^{\prime}$ must be due to microsegregation of alloying elements. Indeed it has been shown that the elements $\mathrm{Al}, \mathrm{Co}, \mathrm{Cr}$, Ta and $\mathrm{Ti}$, for example, enrich in the liquid phase (partition coefficient $k$ less than unity), while Re and $\mathrm{W}$ segregate to the growing $\gamma$ phase ( $k$ greater than unity). ${ }^{[2-27]}$ Thermodynamic databases show the same trends, while allowing more detailed analysis of temperature and composition dependency of the partition coefficient.

Segregation of alloying elements results in solidification under Scheil like conditions, with the interdendritic liquid being mixed by diffusion; back-diffusion in the solid is too slow to achieve equilibrium solidification during technical solidification processing. As the $\gamma^{\prime}$ stabilizing elements- $\mathrm{Al}, \mathrm{Ta}, \mathrm{Ti}-$ segregate to the liquid, interdendritic $\gamma^{\prime}$ will form. The formation of as-cast microstructure and microsegregation has been simulated using 1D models like DICTRA and 2D/3D methods like phase-field. ${ }^{[11,12,28,29]}$ Classically microsegregation models - especially in 1D - use the secondary dendrite arm spacing (SDAS) as the characteristic length. This provides a better approximation to back diffusion as the SDAS is shorter than the primary dendrite arm spacing (PDAS). On the other hand calculated microstructures of single crystal superalloys serve as input to solution heat treatment models. Here the PDAS is more relevant, as homogeneity across the entire primary dendritic length scale needs to be achieved. The two approaches can be joined by using a 2D simulation domain. The 2D domain represents a $2 \mathrm{D}$, isothermal section through the growing dendrite. The PDAS and cooling rate are calculated from the thermal gradient and solidification rate. The PDAS determines the size of the simulation domain and the growing dendrite can develop side arms, hence the backdiffusion distance approaches the SDAS. Both characteristic length scales are therefore included in the model, which is also referred to as the unit-cell model. ${ }^{[12,30]}$ The cooling conditions and initial temperature within the simulation

Table 1 Alloy composition in wt.\%

\begin{tabular}{|c|c|c|c|c|c|c|c|c|c|c|}
\hline Alloy & $\mathbf{N i}$ & Al & Co & $\mathrm{Cr}$ & Mo & $\mathbf{T a}$ & $\mathbf{T i}$ & $\operatorname{Re}$ & $\mathbf{W}$ & Ref \\
\hline CMSX-4 & Bal. & 5.6 & 9.6 & 6.5 & 0.6 & 6.5 & 1.0 & 3.0 & 6.4 & 44 \\
\hline CMSX-10 & Bal. & 5.7 & 3.0 & 2.0 & 0.4 & 8.0 & 0.2 & 6.0 & 5.0 & 1 \\
\hline Model alloy & Bal. & 5.8 & $\cdots$ & 9.0 & $\ldots$ & 7.9 & $\ldots$ & $\ldots$ & 8.8 & 12 \\
\hline
\end{tabular}



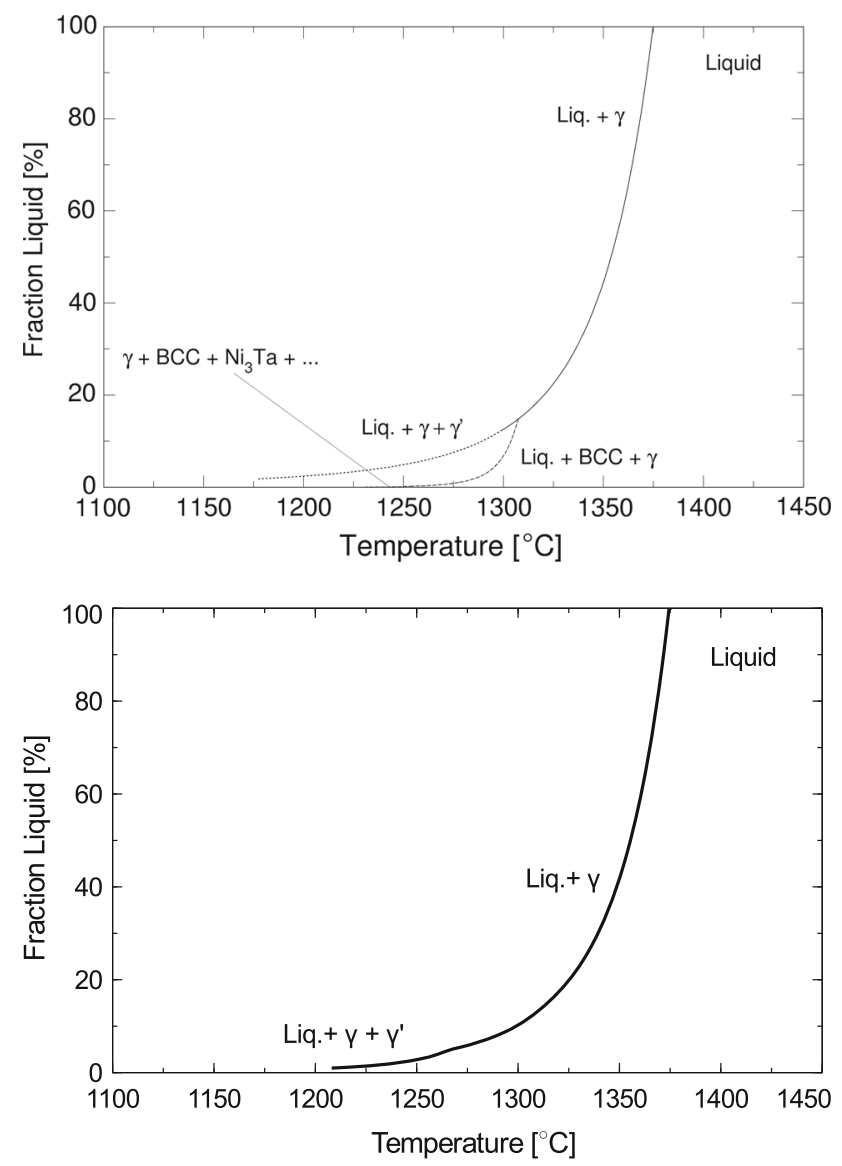

Fig. 1 Fraction liquid as function of temperature for a five component model alloy as obtained from two different thermodynamic databases

domain are then determined from the processing conditions, as defined by the thermal gradient and solidification rate. The microstructure evolution in the $2 \mathrm{D}$ model can then be simulated using a phase-field model coupled to CALPHAD type calculations, allowing to include the appropriate databases for thermodynamic and kinetic data. More details on the phase-field approach and the coupling to thermodynamic and kinetic calculations can be found elsewhere. ${ }^{[31,32]}$

Shown here are results for two single crystal alloys, which show the characteristic microsegregation patterns found in this type of alloys. Results obtained from phasefield simulations using the 2D unit-cell model of directional solidification of superalloys can be seen in Fig. 3. The top row shows simulation results for the five-component model superalloy, whose composition is given in Table 1, while the bottom row shows microsegregation maps obtained from experiments for comparison. Both microstructures show dendrites of $\gamma$ phase, which grow during primary solidification, and interdendritic $\gamma^{\prime}$, which formed during secondary solidification. The microsegregation profiles along the secondary dendrite arms (e.g. [010]) differ from the profiles between secondary arms (e.g. [011]). This kind of pattern had also be described by Ma and Grafe. ${ }^{[33]}$ In this alloy the secondary $\gamma^{\prime}$ is preferentially located between the dendrite

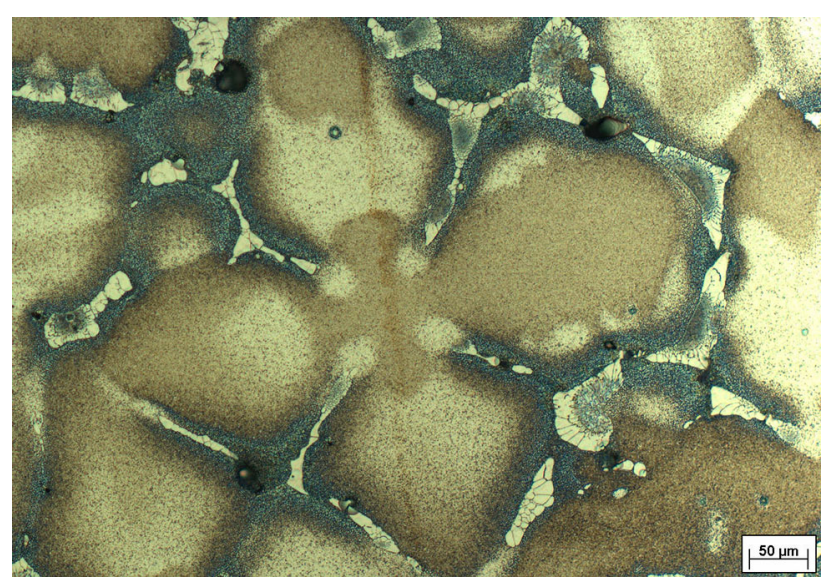

Fig. 2 Typical cross section of directionally solidified CMSX-4, showing primary $\gamma$ dendrites and interdendritic $\gamma^{\prime}$

arms, as seen in both simulation and experiment. It can also be easily seen that during primary solidification, the elements $\mathrm{Al}$ and $\mathrm{Ta}$ segregate heavily to the liquid - partition coefficients $<1$-while $\mathrm{W}$ segregates to the growing dendrites $(k>1)$. The fourth alloying element $\mathrm{Cr}$ shows rather weak segregation to the liquid during primary solidification, with a partition coefficient $\mathrm{k}$ just below one. During the growth of interdendritic $\gamma^{\prime}$ the elements $\mathrm{Al}$ and Ta segregate towards $\gamma^{\prime}$, while $\mathrm{W}$ is rejected. The three elements $\mathrm{Al}, \mathrm{Ta}$ and $\mathrm{W}$ show therefore a monotonic trend from a minimum $(\mathrm{Al}, \mathrm{Ta})$ or maximum $(\mathrm{W})$ concentration at the dendrite core and a monotonous increase/decrease of concentration towards the interdendritic $\gamma^{\prime}$. Cr on the other hand is rejected by both the $\gamma$ and $\gamma^{\prime}$ phase, hence the $\mathrm{Cr}$ minimum concentration is found in the interdendritic $\gamma^{\prime}$ and the maximum concentration in the $\gamma$ phase next to it. This is a peculiar segregation pattern which is widely observed in single crystal superalloys. For example, Fig. 4 shows simulation results for the commercial single crystal superalloy CMSX $-10^{\circledR}$. Here the elements $\mathrm{Al}, \mathrm{Ta}, \mathrm{W}$ and $\mathrm{Cr}$ show the same segregation patterns as in the model alloy in Fig. 3. The segregation behavior of the other alloying elements can be classified as being of the $\mathrm{Al}$ type (Al, Ta, Ti), the $\mathrm{W}$ type $(\mathrm{Re}, \mathrm{W})$ or the Cr type (Cr, Co, Mo). This general trend and the segregation pattern surrounding interdendritic $\gamma^{\prime}$ have also been reported of other alloys. ${ }^{[34,35]}$ The actual values of the partition coefficients however do depend on the overall alloy composition as shown by Hobbs et al. ${ }^{[26]}$ and attempts have been made to minimize microsegregation through additions of for example $\mathrm{Ru}^{[36]}$ The weak partitioning of $\mathrm{Cr}$ during the early stages of solidification has led to some surprising findings however. During a study on CMSX-4 it was found that $\mathrm{Cr}$ showed a partition coefficient greater than one, contrary to what was expected from thermodynamics calculations and simulations. ${ }^{[37]}$ This phenomenon cannot be explained by solid-state diffusion in the $\gamma$ dendrites, as back diffusion would bring the partition coefficient closer to one, but not 'switch' from less to greater to one (or vice versa). Solid state nucleation of $\gamma^{\prime}$ particles inside the $\gamma$ dendrites during cooling after solidification is the key to 

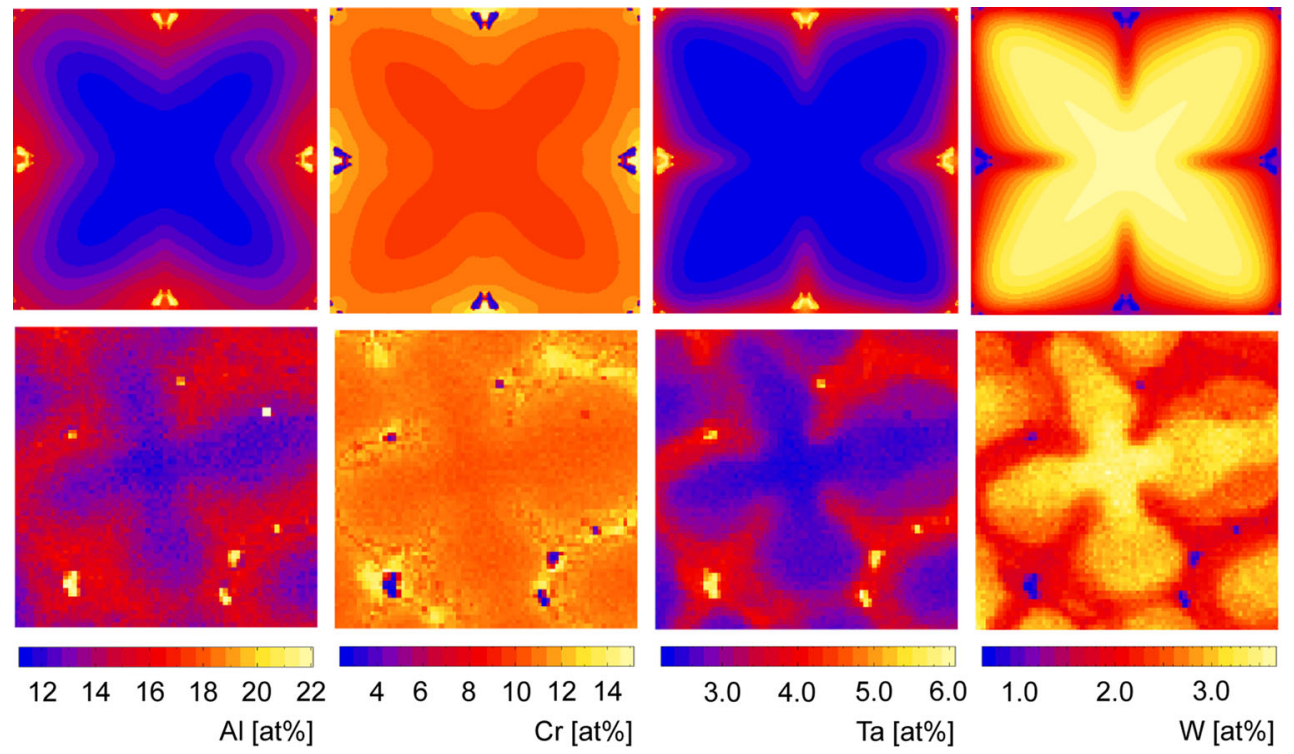

Fig. 3 Results from phase-field simulations (top row) in comparison to micosegregation patterns obtained using WDS on directionally solidified model superalloy ${ }^{[12]}$

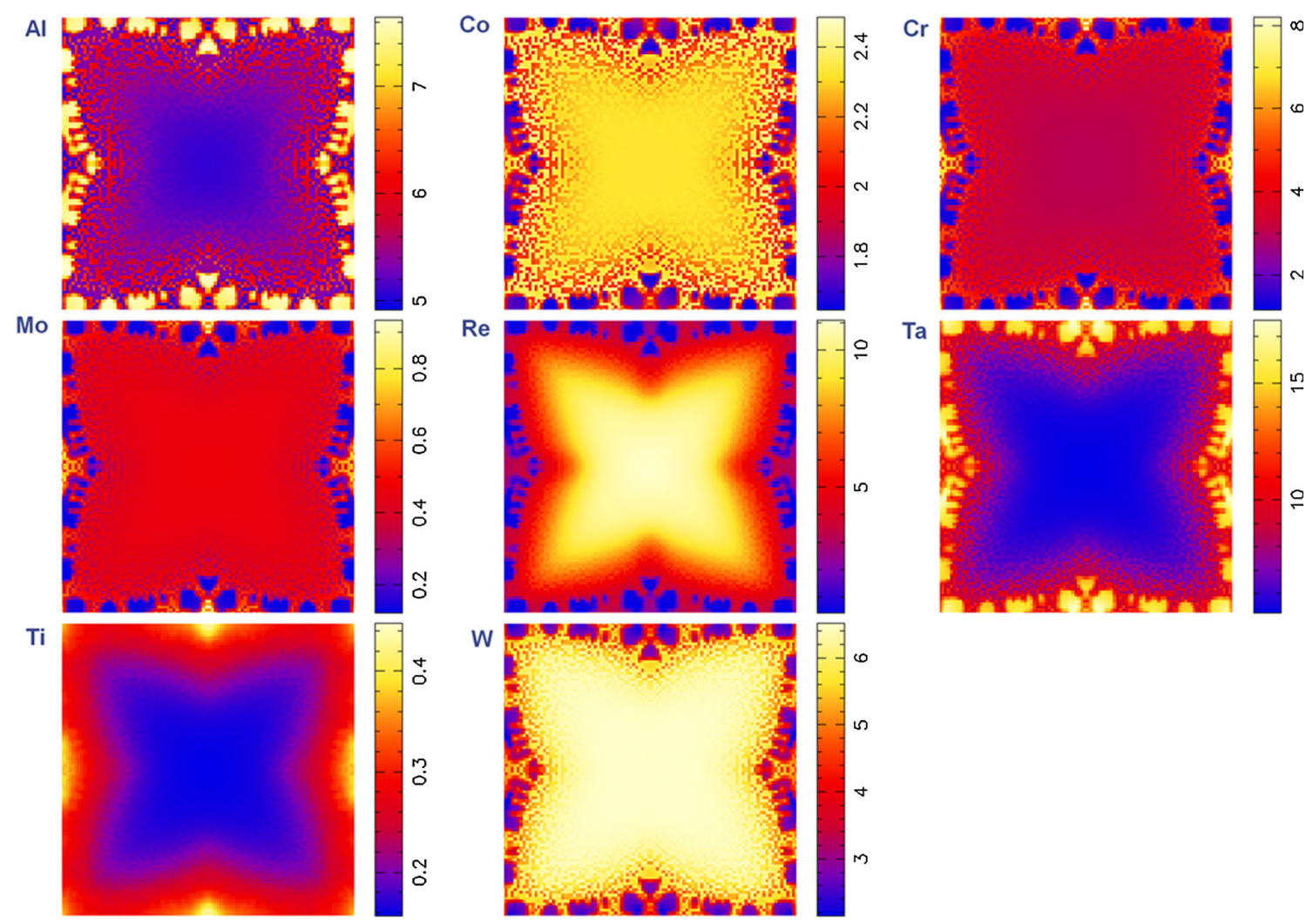

Fig. 4 Microsegregation patterns and phase arrangement calculated for simulated solidification of CMSX-10N

understanding this phenomenon. The simulations shown in Fig. 4 try to mimic this. As can be seen, the interdendritic $\gamma^{\prime}$ is surrounded by speckled areas, with random like appearance. In these areas $\gamma^{\prime}$ has nucleated and grown following a solid state reaction. As the solubility of alloying elements within $\gamma^{\prime}$ particles is very different from the one in the $\gamma$ matrix, segregation on the scale of the $\gamma^{\prime}$ particles occurs, leading to strong variation of the local composition. In the 


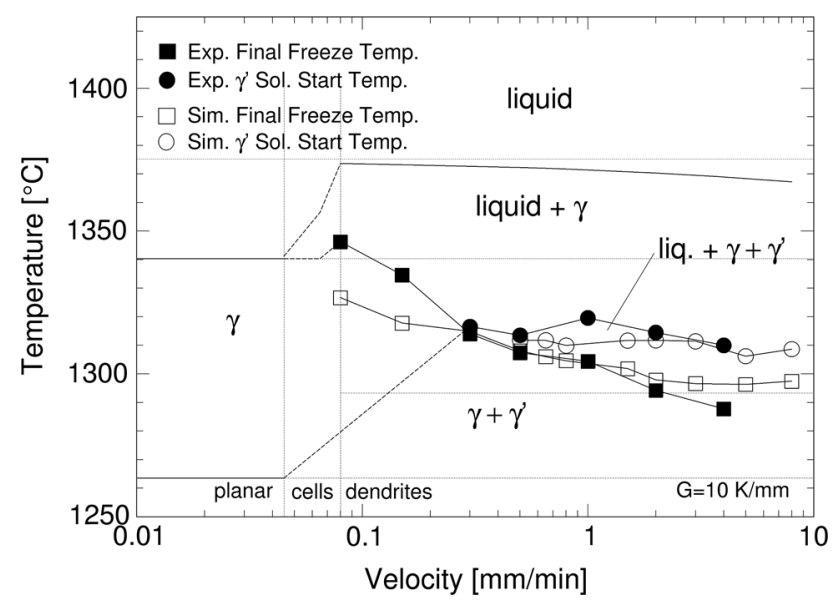

Fig. 5 Sequence of phase formation and approximate transition temperatures as function of solidification rate for steady directional solidification of the five component model superalloy ${ }^{[12]}$

case of elements with partitioning during primary solidification - such as $\mathrm{Cr}$ - this variation can be much stronger than the actual microsegregation from solidification. During energy dispersive spectroscopy (EDS) or wavelength dispersive spectroscopy (WDS) measurements the activation volume of the electron beam is of similar size as the $\gamma^{\prime}$ particles, hence the measurements show a lot of scatter. This makes measurement of microsegregation patterns in single crystal superalloys quite difficult. ${ }^{[38,39]}$ It can be seen that the general segregation behavior and pattern formation is reasonably well understood, but the analysis is less straight forward than it would appear on first glance.

\section{Solidification Sequence}

It is very common to observe that different phases forming during finite cooling rate solidification than during equilibrium solidification; single crystal superalloys are no exception. It is therefore interesting to study how the sequence of phase formation - the solidification sequence - depends on processing conditions, namely the rate of solidification and the thermal gradient.

As single crystal superalloys are designed to show a single phase $\gamma$ region at high temperatures, commonly referred to as the solution heat treatment window, the equilibrium solidification sequence is:

$$
\text { Liq } \rightarrow \text { liq }+\gamma \rightarrow \gamma \rightarrow \gamma+\gamma_{\text {solidstate }}^{\prime}
$$

For technically relevant solidification conditions the following phase formation sequence is commonly observed:

Liq $\rightarrow$ liq $+\gamma \rightarrow$ liq $+\gamma+\gamma^{\prime} \rightarrow \gamma+\gamma^{\prime} \rightarrow \gamma+\gamma^{\prime}+\gamma_{\text {solidstate }}^{\prime}$

How this transition depends on the solidification rate during directional solidification is illustrated in Fig. 5. This diagram shows the sequence of phase formation with the

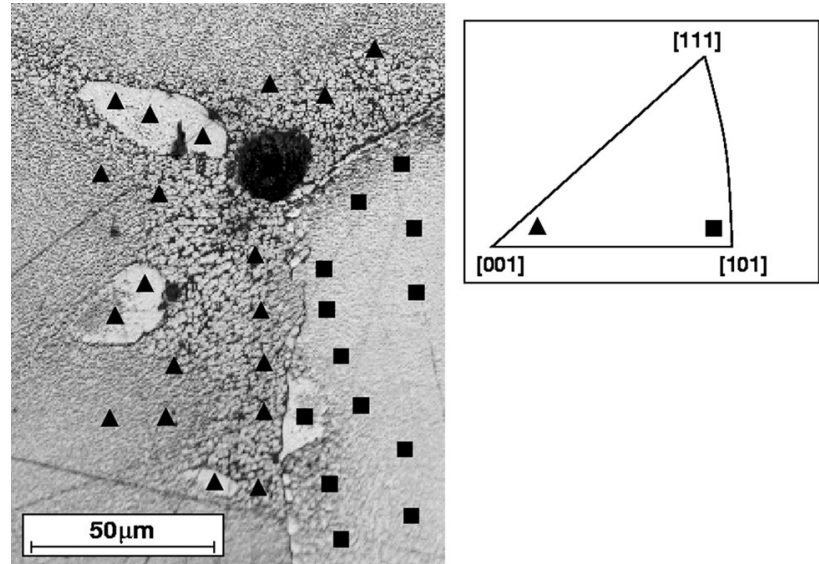

Fig. 6 EBSD measurement of the interdendritic $\gamma^{\prime}$ orientation along a grain boundary. Directionally solidified 5 component model alloy ${ }^{[42]}$

approximate temperatures at which transitions occur, as function of the steady state solidification rate during directional solidification. The diagram in Fig. 5 was determined for the five component model superalloy. Additional details of the experimental procedure and simulations can be found elsewhere. ${ }^{[12]}$ It must be noted that this diagram is only valid for steady state solidification and for a thermal gradient of $10 \mathrm{~K} / \mathrm{mm}$. Closed symbols mark results obtained from experiments, open symbols simulation results. Experimental results were obtained from longitudinal sections of directionally solidified and quenched samples, while simulations were performed using the 2D unit-cell model. Furthermore, full lines without symbols were obtained from analytical models. Heavy dashed lines are merely guides to the eye which are filling in gaps by connecting full lines, while thin dashed lines indicate transition between solidification regimes or transition temperatures obtained from equilibrium calculations. Three solidification regimes are observed, planar, cellular and dendritic. Planar solidification occurs at low solidification rates, which changes to cellular solidification as the solidification rate increases. In both cases liquid transforms into solid $\gamma$, with a liq $+\gamma$ two phase region being observed for cellular solidification. The relevant transitions were also obtained experimentally. At the lower end of solidification rates within the dendritic regime, liquid still transforms into $\gamma$ phase, with no appearance of $\gamma^{\prime}$. The latter only forms at sufficiently high solidification rates. Interestingly the solidification interval, as defined by the temperature difference between the onset of solidification and the temperature at the end of solidification, increases as the solidification rate increases. The temperature at which $\gamma^{\prime}$ nucleates on the other hand is not affected by the solidification rate. This behavior is observed for both, experiments and simulation. Scheil calculations predict $\gamma^{\prime}$ formation approximately $10 \mathrm{~K}$ below the phasefield simulation results (thin horizontal dashed line at $1293{ }^{\circ} \mathrm{C}$ ). Similar observation were made from simulations of solidification in CMSX-4. ${ }^{[28]}$ This is a strong indication of the importance of backdiffusion of alloying elements 
during solidification and in agreement with the findings elsewhere. ${ }^{[40]}$

\section{Formation of Interdendritic $\gamma^{\prime}$}

The formation of interdendritic $\gamma^{\prime}$, or the $\gamma / \gamma^{\prime}$ eutectic, as it is often referred to, is a distinct feature of single crystal superalloy, as-cast microstructures. It has been demonstrated recently that it can initiate recrystallization during heat treatment. $^{[41]}$ As parts made from these alloys are meant to be single crystal, it is needless to say that the appearance of recrystallization is unwanted and leads to scrapping of parts.

It is of interest to take a look into how the interdendritic $\gamma^{\prime}$ forms, and to study the final stages of solidification in more detail. It has been shown by analyzing the nucleation barrier for $\gamma^{\prime}$ nucleation, that $\gamma^{\prime}$ nucleates from the $\gamma$ phase through a solid state reaction. ${ }^{[8]}$ This is energetically favorable due to the low interfacial energy between $\gamma$ and $\gamma^{\prime}$, which do form a coherent interface. If this nucleation takes place close to the solid-liquid interface, $\gamma^{\prime}$ can grow

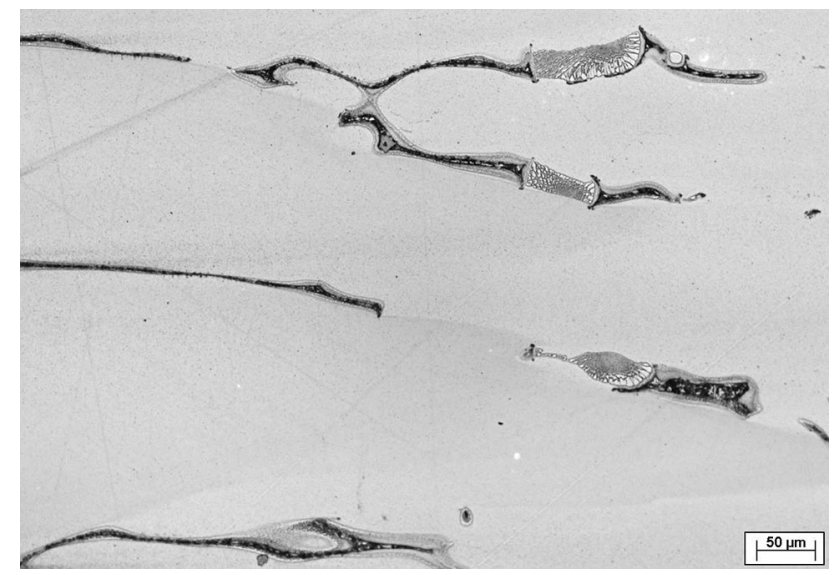

Fig. 7 Longitudinal section of directionally solidified and quenched CMSX-4 sample, showing interdendritic $\gamma^{\prime}$ growing from $\gamma$ dendrites into the surrounding liquid. Hence the interdendritic $\gamma^{\prime}$ has the same crystallographic orientation as at least one of the adjacent dendrites. ${ }^{[8,35]}$ Figure 6 shows Electron Backscatter Diffraction (EBSD) measurements on interdendritic $\gamma^{\prime}$ and surrounding dendrites located on a grain boundary. ${ }^{[42]}$ The two different orientations are marked using squares and triangles respectively. It is seen that the $\gamma^{\prime}$ particle inherits the orientation of one of the two grains.

The $\gamma^{\prime}$ islands exhibit a structure within themselves, as seen in Fig. 2. It consists of a small scale, almost checker board like part, and a larger fan-like part. Both parts are usually attached to a dendrite. Some controversy did exist on how these structures form. Earlier works proposed the fan-like structure forms first, with the finer structure nucleating from the melt. ${ }^{[43]}$ This seems rather unlikely, as this would require very high undercooling and an extremely large number of nuclei in the liquid pockets. Given the nature of the solid state nucleation mechanism of the $\gamma^{\prime}$ phase, it is more likely that the fine structure forms first from a large number of solid state nuclei, which then fan out into the remaining liquid. The structures seen in Fig. 7 show a longitudinal section of a directionally solidified and quenched sample. The large white areas show the $\gamma$ dendrites, with the interdendritic $\gamma^{\prime}$ attached to it. Quenched liquid appears at rather elongated, almost black regions. These structures support the hypothesis of the fine structure forming first; here the fine structure fans out into the quenched liquid. A more rigorous study confirm this even further, with the aid of focus-ion beam (FIB), EBSD and EDS analyses. ${ }^{[35]}$

The actual growth process of the interdendritic $\gamma^{\prime}$ is shown in Fig. 8, as a result of a phase-field simulation for the 5 component model alloy. The simulation has been simplified as only a single nucleation site for $\gamma^{\prime}$ is taken into account. The fine and the fanned structures of the interdendritic $\gamma^{\prime}$ are thus omitted. The simulation was done using the thermodynamic database developed by Dupin, which models the $\gamma / \gamma^{\prime}$ reaction in the Ni-Al system as a peritectic one. ${ }^{[22]}$ After nucleation the particle initially growths rapidly at the expense of the $\gamma$ phase. Typical grooves are formed at the liquid $/ \gamma / \gamma^{\prime}$ triple points. Towards the end both phases $-\gamma$ and $\gamma^{\prime}$-grow. Figure 9 shows the same simulation, for the same alloy, using the TTNi $7^{[23]}$ thermody-
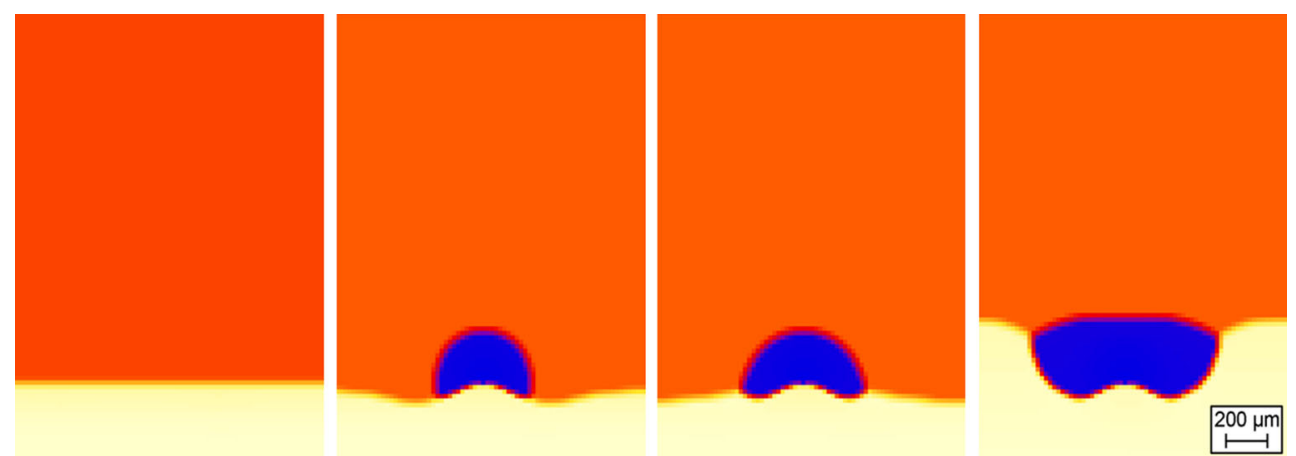

Fig. 8 Growth of interdendritic $\gamma^{\prime}$ in the five component model alloy, calculated using phase-field coupled to a thermodynamic database, using a peritectic description of the $\mathrm{Ni}-\mathrm{Al}$ system $^{[8]}$ 

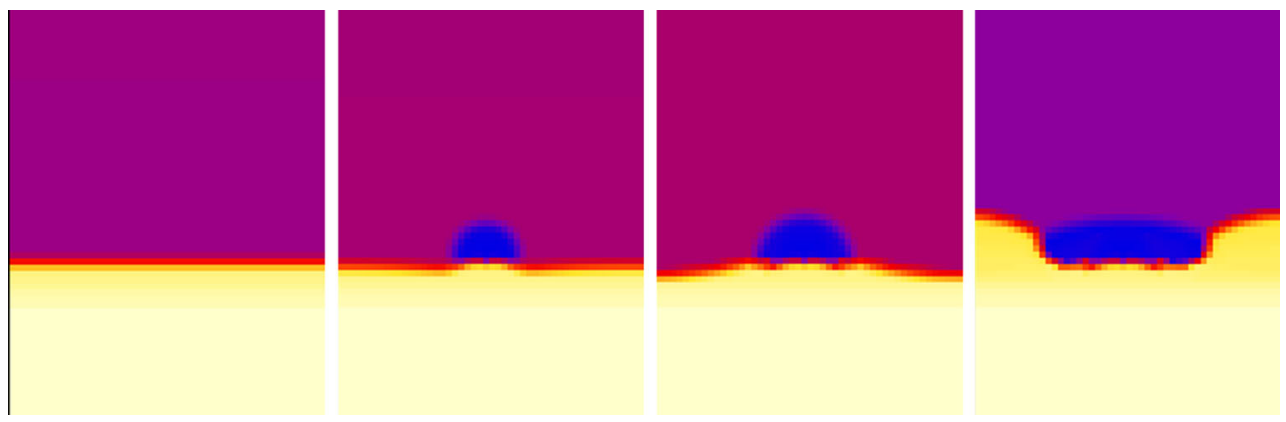

Fig. 9 Growth of interdendritic $\gamma^{\prime}$ in the five component model alloy, calculated using phase-field coupled to a thermodynamic database, using a eutectic description of the $\mathrm{Ni}-\mathrm{Al}$ system

namic database, which models the $\gamma / \gamma^{\prime}$ reaction as a eutectic one. Interestingly the result is qualitatively the same, the same pattern and morphology is observed. More work is needed to draw final conclusion from this, but it might be save to say that the distinction whether the reaction is a peritectic or eutectic one is not straight forward in this particular case. At least it cannot be made on the nature of the Ni-Al binary alone. The neutral term interdendritic $\gamma^{\prime}$ seems therefore appropriate.

\section{Summary and Conclusion}

The formation of as-cast microstructures in single crystal superalloys has been widely studied using experimental and numerical techniques. This includes elemental segregation, alloy constitution and phase-formation. Advances in the availability of thermodynamic and diffusion data, combined with modern modeling and simulation tools allow supplementing experiments with simulations which take the full compositional complexity of these alloys into account. As a consequence the sequence of phase formation and microsegregation behavior of the alloying elements is reasonable well understood, at least for solidification in regimes typical for directional solidification. Although fully quantitative predictions remain a challenge, modern simulations tools are capable of handling the full compositional complexity of these alloys.

The agreement between experiments and simulations for microstructure formation during solidification of single crystal superalloys indicates that contemporary modeling methods and the all-important thermodynamic and diffusion data have reached a decent state of maturity. Indeed simulation results as presented here have been used to derive a method to optimize solution heat treatments of these alloys. ${ }^{[11]}$ With this method the incipient melting temperature is determined from calculated microstructures. This information is then used to control the temperature in the simulation, allowing it only to increase as the microstructure homogenizes.

A few challenges remain, such as the never ending quest for improved thermodynamic and kinetic data for multicomponent alloys. The formation of undesired phases, such as TCP phases, or carbides still prove a challenge to simulation tools, due to their more complex morphology. The increasing use of additive manufacture techniques, requires revisiting some of the understanding of the solidification path in single crystal superalloys, as these processes show significantly higher cooling rates. The already established knowledge however provides a solid basis.

\section{Open Access}

This article is distributed under the terms of the Creative Commons Attribution 4.0 International License (http:// creativecommons.org/licenses/by/4.0/), which permits unrestricted use, distribution, and reproduction in any medium, provided you give appropriate credit to the original author(s) and the source, provide a link to the Creative Commons license, and indicate if changes were made.

\section{References}

1. R.C. Reed, The Superalloys, Cambridge University Press, Cambridge, 2006

2. R.C. Reed, T. Tao, and N. Warnken, Alloys-by-Design: Application to Nickel-Based Single Crystal Superalloys, Acta Mater, 2009, 57(19), p 5898-5913

3. C. Rae, Alloys by Design: Modelling Next Generation Superalloys, Mater. Sci. Technol., 2009, 25, p 479-487

4. M. Durand-Charre, The Microstructure of Superalloys, Gordon and Breach Science Publishers, Amsterdam, 1997

5. C.T. Sims, N.S. Stoloff, and W.C. Hagel, Superalloys II, Wiley, New York, 1987

6. N.S. Husseini, D.P. Kumah, J.Z. Yi, C.J. Torbet, D.A. Arms, E.M. Dufresne, T.M. Pollock, J. Wayne Jones, and R. Clarke, Mapping Single-Crystal Dendritic Microstructure and Defects in Nickel-Base Superalloys with Synchrotron Radiation, Acta Mater., 2008, 56(17), p 4715-4723

7. D. $\mathrm{Ma}$ and P.R. Sahm, Investigation on Solidification Processing of the Directionally Solidified Superalloy CMSX 6, Z. Metallkd., 1995, 86(11), p 764-768

8. N. Warnken, D. Ma, M. Mathes, and I. Steinbach, Investigation of Eutectic Island Formation in SX Superalloys, Mater. Sci. Eng., A, 2005, 413-414, p 267-271

9. S. Tin and T.M. Pollock, Phase Instabilities and Carbon Additions in Single-Crystal Nickel-Base Superalloys, Mater. Sci. Eng., A, 2003, 348(1-2), p 111-121 
10. C.M.F. Rae, M.S.A. Karunaratne, C.J. Small, R.W. Broomfield, C.N. Jones, and R.C. Reed, Topologically Close Packed Phases in an Experimental Rhenium-Containing Single Crystal Superalloy, Superalloys 2004. Minerals, Metals $\ \&$ Materials Soc, 2000

11. N. Warnken, H. Larsson, and R.C. Reed, Coupled Modelling of Solidification and Solution Heat Treatment of Advanced Single Crystal Nickel Base Superalloy, Mater. Sci. Technol., 2009, 25(2), p 179-185

12. N. Warnken, D. Ma, A. Drevermann, R.C. Reed, S.G. Fries, and I. Steinbach, Phase-Field Modelling of As-cast Microstructure Evolution in Nickel-Based Superalloys, Acta Mater., 2009, 57(19), p 5862-5875

13. F. Cosentino, N. Warnken, J.-C. Gebelin, and R.C. Reed, Numerical and Experimental Study of Post-Heat Treatment Gas Quenching and Its Impact on Microstructure and Creep in CMSX-10 Superalloy, J. Mater. Process. Technol., 2013, 213(12), p 2350-2360

14. G.E. Fuchs, Solution Heat Treatment Response of a Third Generation Single Crystal Ni-Base Superalloy, Mater. Sci. Eng., A, 2001, 300(1-2), p 52-60

15. P. Auburtin, S.L. Cockcroft, A. Mitchell, and T. Wang, Freckle Formation in Superalloys, Superalloys 2000, TMS, 2000

16. C. Beckermann, J.P. Gu, and W.J. Boettinger, Development of a Freckle Predictor via Rayleigh Number Method for SingleCrystal Nickel-Base Superalloy Castings, Metall. Mater. Trans. A, 2000, 31(10), p 2545-2557

17. S.M. Copley, A.F. Giamei, S.M. Johnson, and M.F. Hornbeck, Origin of Freckles in Unidirectionally Solidified Castings, Metallurgical transactions., 1970, 1, p 2193

18. K. Hilpert, D. Kobertz, V. Venugopal, M. Miller, H. Gerads, F.J. Bremer, and H. Nickel, Phase-Diagram Studies on the AlNi System, Z. Naturforsch. A: Phys. Sci., 1987, 42(11), p $1327-1332$

19. J.H. Lee and J.D. Verhoeven, Peritectic Formation in the Ni-Al System, J. Cryst. Growth, 1994, 144(3-4), p 353-366

20. O. Hunziker and W. Kurz, Directional Solidification and Phase Equilibria in the Ni-Al System, Metall. Mater. Trans. A, 1999, 30(12), p 3167-3175

21. I. Ansara, N. Dupin, H.L. Lukas, and B. Sundman, Thermodynamic Assessment of the Al-Ni System, J. Alloy. Compd., 1997, 247(1-2), p 20-30

22. N. Dupin and B. Sundman, A Thermodynamic Database for Ni-Base Superalloys, Scand. J. Metall., 2001, 30(3), p 184192

23. N. Saunders, Phase Diagram Calculations for Ni-Based Superalloys, Superalloys 1996, 1996

24. W. Boettinger and U. Kattner, Analysis of Solidification Path and Microsegregation in Multicomponent Alloys, Modelling of Casting, Welding and Advanced Solidification Processes VIII, 1998

25. S.N. Tewari, M. Vijayakumar, J.E. Lee, and P.A. Curreri, Solutal Partition Coefficients in Nickel-Based Superalloy PWA-1480, Mater. Sci. Eng., A, 1991, 141(1), p 97-102

26. R. Hobbs, S. Tin, and C. Rae, A Castability Model Based on Elemental Solid-Liquid Partitioning in Advanced Nickel-Base Single-Crystal Superalloys, Metallurgical and Materials Transactions A, 2005, 36(10), p 2761-2773

27. P.K. Sung and D.R. Poirier, Liquid-Solid Partition Ratios in Nickel-Base Alloys, Metall. Mater. Trans. A, 1999, 30(8), p 2173-2181

28. C. Walter, B. Hallstedt, and N. Warnken, Simulation of the Solidification of CMSX-4, Mater. Sci. Eng. A, 2005, 397(1-2), p 385-390
29. M.S.A. Karunaratne, D.C. Cox, P. Carter, and R.C. Reed. Modelling of the Microsegregation in CMSX-4 Superalloy and Its Homogenisation During Heat Treatment, Superalloys 2000, TMS, 2000

30. I. Lopez-Galilea, S. Huth, S. Fries, N. Warnken, I. Steinbach, and W. Theisen, Microsegregation and Secondary Phase Formation During Directional Solidification of the SingleCrystal Ni-Based Superalloy LEK94, Metall. Mater. Trans. A, 2012, 43(13), p 5153-5164

31. I. Steinbach, B. Böttger, J. Eiken, N. Warnken, and S.G. Fries, CalPhaD and PhaseField Modeling: A Successful Liaison, $J$. Phase Equilib. Diffus., 2007, 28(1), p 101-106

32. J. Eiken, B. Bottger, and I. Steinbach, Multiphase-Field Approach for Multicomponent Alloys with Extrapolation Scheme for Numerical Application, Phys. Rev. E, 2006, 73(6), p 066122-066129

33. D. Ma and U. Grafe, Microsegregation in Directionally Solidified Dendritic-Cellular Structure of Superalloy CMSX4, Mater. Sci. Eng., A, 1999, 270(2), p 339-342

34. N. Warnken, D. Ma, A. Drevermann, I. Steinbach, and S.G. Fries, Quantitative Simulations of Microstructure Evolution in Single Crystal Superalloys During Solution Heat Treatment, Int. Heat Treat. Surf. Eng., 2009, 3, p 40-44

35. A. Heckl, R. Rettig, S. Cenanovic, M. Göken, and R.F. Singer, Investigation of the Final Stages of Solidification and Eutectic Phase Formation in $\mathrm{Re}$ and $\mathrm{Ru}$ Containing Nickel-Base Superalloys, J. Cryst. Growth, 2010, 312(14), p 2137-2144

36. R.M. Kearsey, J.C. Beddoes, P. Jones, and P. Au, Compositional Design Considerations for Microsegregation in Single Crystal Superalloy Systems, Intermetallics, 2004, 12(7-9), p 903-910

37. M.S.A. Karunaratne, D.C. Cox, P. Carter, and R.C. Reed. Modelling of the Microsegregation in CMSX-4 Superalloy and Its Homogenisation During Heat Treatment, Superalloys 2000, TMS

38. N. D’Souza, R. Beanland, C. Hayward, and H.B. Dong, Accuracy of Composition Measurement Using X-ray Spectroscopy in Precipitate-Strengthened Alloys: Application to Ni-Base Superalloys, Acta Mater, 2011, 59(3), p 1003-1013

39. N. D'Souza, M. Lekstrom, and H.B. Dong, An Analysis of Measurement of Solute Segregation in Ni-Base Superalloys using X-ray Spectroscopy, Mater. Sci. Eng., A, 2008, 490(1-2), p 258-265

40. A. Thirumalai, A. Akhtar, and R.C. Reed, Extent of Back Diffusion During Solidification of Experimental Nickel Based Single Crystal Superalloy, Mater. Sci. Technol., 2006, 22(1), p $1-13$

41. L. Wang, F. Pyczak, J. Zhang, L.H. Lou, and R.F. Singer, Effect of Eutectics on Plastic Deformation and Subsequent Recrystallization in the Single Crystal Nickel Base Superalloy CMSX-4, Mater. Sci. Eng., A, 2012, 532, p 487-492

42. N. Warnken, Simulation of Microstructure Formation during Solidification and Solution Heat Treatment of a Novel Single Crystal Superalloy, RWTH-Aachen, Foundry Institute, Aachen, 2007

43. N. D'Souza and H.B. Dong, Solidification Path in ThirdGeneration Ni-Based Superalloys, with an Emphasis on Last Stage Solidification, Scr. Mater., 2007, 56(1), p 41-44

44. K. Harris, G.L. Erickson, S.L. Sikkenge, W.D. Brentnall, J.M. Aurrecoechea, and K.G. Kubarych. Development of Rhenium Containing Superalloys CMSX-4 and CM 186 LC for Single Crystal Blade and Directionally Solidified Vane Applications in Advanced Turbine Engines, Seventh International Symposium on Superalloys, 1992 (Seven Springs), S.D. Antolovich 